Ann. Biol. anim. Bioch. Biophys., 1978, 18 (6), 1293-1308.

\title{
Action de la fluorescéine, de l'alizarine, du bleu de calcéine et de diverses doses de tétracycline sur la croissance de la truite et de la carpe
}

par F. J. MEUNIER, G. BOIVIN *

\author{
Equipe de Recherche « Formations squelettiques》, \\ Laboratoire d'Anatomie comparée \\ Université Paris 7 , \\ 2, Place Jussieu, 75221 Paris Cedex 05, France. \\ * Institut de Morphologie, Ecole de Médecine 1211 \\ Genève 4, Suisse.
}

Summary. Action of fluorescent markers on growth of trout and carp. Effects of different doses of fluorescein, alizarin, calcein blue and tetracycline.

The doses of some fluorescent markers $(50 \mathrm{mg} / \mathrm{kg}$ of tetracycline chlorhydrate, $40 \mathrm{mg} / \mathrm{kg}$ of fluorescein, $80 \mathrm{mg} / \mathrm{kg}$ of alizarin red $S$ and $30 \mathrm{mg} / \mathrm{kg}$ of calcein blue) required to obtain good labelling of bone tissue were found to be compatible with optimal growth of trout and carp, and to not affect weight gain. There was no mortality due to fluorochromes. These markers can thus be confidently used, within the physiological values of this report, to study the bone growth and biodynamics of these fishes. However, the present study did not explain some effects of the markers on bone mineralization or their localized effect on skeletal tissues («calciotraumatic lines »). Injections of high pharmacological doses of tetracycline $(500 \mathrm{mg} / \mathrm{kg}$ ) resulted in high trout and carp mortality ; a significant decrease in weight gain was observed in the surviving fishes. The particularly dramatic symptoms preceding the death of the carp suggest that tetracycline affects both physiology of the liver and the water balance in this species. Results also showed that carp were more sensitive than trout to the effects of large doses of tetracycline.

La tétracycline, la fluorescéine, l'alizarine et le bleu de calcéine sont des substances qui sont retenues in vivo, de façon durable, dans les tissus squelettiques en cours de minéralisation (Milch ef al., 1957 ; Suzuki et Mathews, 1966 ; Dhem, 1967 ; Olerud et Lorenzi, 1970 ; Rahn et Perren, 1970 ; Marois et Marois, 1971 ; Vilmann, 1974 ; Skinner et Nalbandian, 1975). Ces marqueurs vitaux sont détectables, sur des préparations histologiques non décalcifiées, par la fluorescence qu'ils émettent lorsqu'ils sont irradiés en lumière ultra-violette (microscopie de fluorescence). L'utilisation de l'une de ces substances ou la combinaison de plusieurs d'entre elles ont permis d'étudier avec succès la dynamique du tissu osseux chez les Mammifères (voir en particulier Dupont, 1964 ; Dhem, 1967 ; Skinner ef Nalbandian, 1975). 
L'utilisation de cette technique dans l'étude de la physiologie du squelette chez les Poissons est peu fréquente (Lopez, 1970 ; Simmons et al., 1970 ; Meunier, 1972 et 1974 ; Meunier et Boivin, 1972 et 1974). Compte tenu des résultats obtenus chez les Mammifères, le «marquage vital » des tissus squelettiques ouvre pourtant des perspectives intéressantes pour l'étude des phénomènes du modelage et du remodelage osseux chez les Poissons. Il est nécessaire de vérifier que l'utilisation de ces substances est compatible avec la vie et la croissance des animaux expérimentés. Des effets nocifs de la tétracycline n'apparaissent sur les Poissons qu'à partir de fortes doses très supérieures à celles suffisantes pour avoir un bon marquage (Weber et Ridgway, 1962 1967 ; de Bont et Van Coillie, 1966 ; Van Coillie, 1967 ; Jones, 1969 ; Kobayashi et al.,, 1964). Il faut toutefois noter que chez Fundulus heteroclitus l'injection d'une faible dose de tétracycline paraît avoir une action inhibitrice sur la minéralisation des écailles en régénération (Bevelander et Goss, 1962).

En ce qui concerne l'alizarine, la fluorescéine ef le bleu de calcéine, nous sommes les seuls, à notre connaissance, à avoir utilisé ces trois produits de façon courante chez des Téléostéens (Meunier, 1974). Aucune étude n'a donc encore été faite sur leur éventuelle action sur la croissance des Poissons.

Tout en poursuivant l'étude de la dynamique osseuse chez la carpe et la truite, il est apparu nécessaire de vérifier si les « marqueurs vitaux » ont une action nocive sur la biologie de ces deux espèces. La présente note relate les résultats relatifs à l'effet des fluorochromes sur la vie ef la croissance pondérale des animaux. Les effets de la tétracycline ont été particulièrement étudiés car cet antibiotique peut avoir une utilisation thérapeutique en Ichthyopathologie.

\section{Matériel et méthodes.}

Les divers fluorochromes testés dans ce travail sont :

- le chlorhydrate de tétracycline $(=\mathrm{CHTC}$ ) fabriqué par Specia (solution à 1 p. 100 dans du $\mathrm{NaCl}$ à 0,6 p. 100);

TABLEAU 1

Répartition des poissons dans choque lot en fonction des différentes doses de fluorochromes

\begin{tabular}{|c|c|c|c|c|c|c|}
\hline \multicolumn{4}{|c|}{ Truites } & \multicolumn{3}{|r|}{ Carpes } \\
\hline & lot & Nbre & fluorochrome & lot & Nbre & fluorochrome \\
\hline 1re série & $\begin{array}{l}\text { A } \\
B \\
C \\
D \\
E\end{array}$ & $\begin{array}{l}5 \\
5 \\
5 \\
6 \\
5\end{array}$ & $\begin{array}{l}\text { témoins } \\
50 \mathrm{mg} / \mathrm{kg} \mathrm{CHTC} \\
100 \mathrm{mg} / \mathrm{kg} \text { CHTC } \\
40 \mathrm{mg} / \mathrm{kg} \text { fluorescéine } \\
80 \mathrm{mg} / \mathrm{kg} \text { alizarine }\end{array}$ & $\begin{array}{l}A \\
B \\
C \\
D \\
E\end{array}$ & $\begin{array}{l}10 \\
11 \\
10 \\
11 \\
11\end{array}$ & $\begin{array}{l}\text { témoins } \\
50 \mathrm{mg} / \mathrm{kg} \mathrm{CHTC} \\
100 \mathrm{mg} / \mathrm{kg} \text { CHTC } \\
500 \mathrm{mg} / \mathrm{kg} \text { CHTC } \\
5 \times 100 \mathrm{mg} / \mathrm{kg} \mathrm{CHTC}\end{array}$ \\
\hline $2^{e}$ série & $\begin{array}{l}F \\
G \\
H\end{array}$ & $\begin{array}{r}9 \\
11 \\
11\end{array}$ & $\begin{array}{l}\text { témoins } \\
5 \times 100 \mathrm{mg} / \mathrm{kg} \mathrm{CHTC} \\
500 \mathrm{mg} / \mathrm{kg} \mathrm{CHTC}\end{array}$ & $\begin{array}{l}F \\
G \\
H \\
I\end{array}$ & $\begin{array}{l}11 \\
11 \\
11 \\
11\end{array}$ & $\begin{array}{l}\text { témoins } \\
80 \mathrm{mg} / \mathrm{kg} \text { alizarine } \\
40 \mathrm{mg} / \mathrm{kg} \text { fluorescéine } \\
30 \mathrm{mg} / \mathrm{kg} \text { bleu de calcéine }\end{array}$ \\
\hline
\end{tabular}


- la fluorescéine ou «calcein 》 (2,7-bis(bis(carboxymethyl)-aminomethyl)-fluorescein) de Merck (solution à 2 p. 100 dans $\mathrm{NaHCO}_{3}$ à 2 p. 100);

- l'alizarine ou « Alizarinrot » de Fluka (solution à 1,6 p. 100 dans $\mathrm{NaCl}$ à 0,6 p. 100);

- le bleu de calcéine ou « Calceinblau » de Fluka (solution à 3 p. 100 dans $\mathrm{NaHCO}_{3}$ à 2 p. 100).

Les doses injectées par voie intrapéritonéale (†abl. 1) ont été choisies selon les recommandations des auteurs ayant mis au point ces méthodes chez les Mammifères (Milch ef al., 1957 ; Suzuki et Mathews, 1966 ; Dhem, 1967 ; Olerud et Lorenzi, 1970 ; Rahn et Perren, 1970).

Cette étude a été faite sur des truitelles (Salmo irideus) de $10 \mathrm{à} 70 \mathrm{~g}$ et sur des carpillons (Cyprinus carpio) de 20 à $94 \mathrm{~g}$. Les poissons, munis, dans l'opercule, d'une broche métallique numérotée (type Présadom), sont soumis à une période d'adaptation de 15 jours. Les truitelles sont élevées dans un bac de 300 litres d'eau à $13^{\circ} \pm 1{ }^{\circ} \mathrm{C}$; elles sont nourries avec des granulés d'aliments artificiels et des vers de vase (larves de chironomes). Les carpillons sont répartis dans 2 bacs de 120 litres d'eau maintenue à $20^{\circ} 5 \pm 1^{\circ} \mathrm{C} *$ et ils sont nourris avec des granulés (deux distributions journalières).

Les différents lots ont été réalisés avec un maximum d'individus, en fonction de nos approvisionnements. Les animaux de la première série de truites ont été répartis au hasard, alors que dans les trois autres séries nous avons essayé de réaliser des lots homogènes tenant compte de l'éventail des tailles (tabl. 1). Les animaux témoins n'ont reçu aucune injection. Les autres ont reçu leur dose de fluorochrome en une seule injection sauf pour les truites du lot $G$ et les carpes du lot $E$ qui ont eu une injection quotidienne de CHTC à raison de $100 \mathrm{mg} / \mathrm{kg}$ et ce durant 5 jours consécutifs.

Les poissons des différents lots de truitelles et de carpillons ont été pesés et mesurés (longueur totale) au début et en fin d'expérience. Le poids a, de plus, été mesuré tous les jours pendant la première semaine de l'expérimentation puis une fois par semaine et en fin d'expérience, soit au bout de deux mois environ.

Pour préciser certains aspects particuliers de l'action de la tétracycline sur la carpe, nous avons étudié : 1) l'action d'une forte dose de CHTC (une injection de $500 \mathrm{mg} / \mathrm{kg}$ sur deux carpillons pesant 52 et $70 \mathrm{~g}$ et maintenus à jeun à $18{ }^{\circ} \mathrm{C} ; 2$ ) pour comparaison, deux carpillons (34,5 et $54,5 \mathrm{~g}$ ) ont reçu un volume équivalent de solvant seul $(\mathrm{NaCl}$ à $0,6 \mathrm{p} .100)$. Ces poissons ont été conservés à $21 \pm 1^{\circ} \mathrm{C}$ et pesés régulièrement. Par ailleurs, pour contrôler l'action du mode opératoire, nous avons injecté un lot de 5 truites $(9$ à $12 \mathrm{~g}$ ) avec un volume de $\mathrm{NaCl}$ à $0,6 \mathrm{p}$. 100, équivalent à celui d'une dose de $50 \mathrm{mg} / \mathrm{kg}$ de CHTC.

Nous avons rassemblé dans les tableaux 2 à 5 les moyennes des poids obtenus à chaque pesée, leurs écarts types et le nombre d'animaux rescapés pour les différents lots.

L'évolution de l'accroissement pondéral (en pourcentage du poids moyen initial) en fonction du temps est représentée sur des graphiques (fig. 1 à 5 ) ; les traits des différentes courbes n'ont pas été tracés lorsque les points sont trop rapprochés, pour ne pas surcharger les figures.

* Cette température se situe dans l'optimum thermique permettant une bonne croissance de cette espèce (Huet, 1962). 


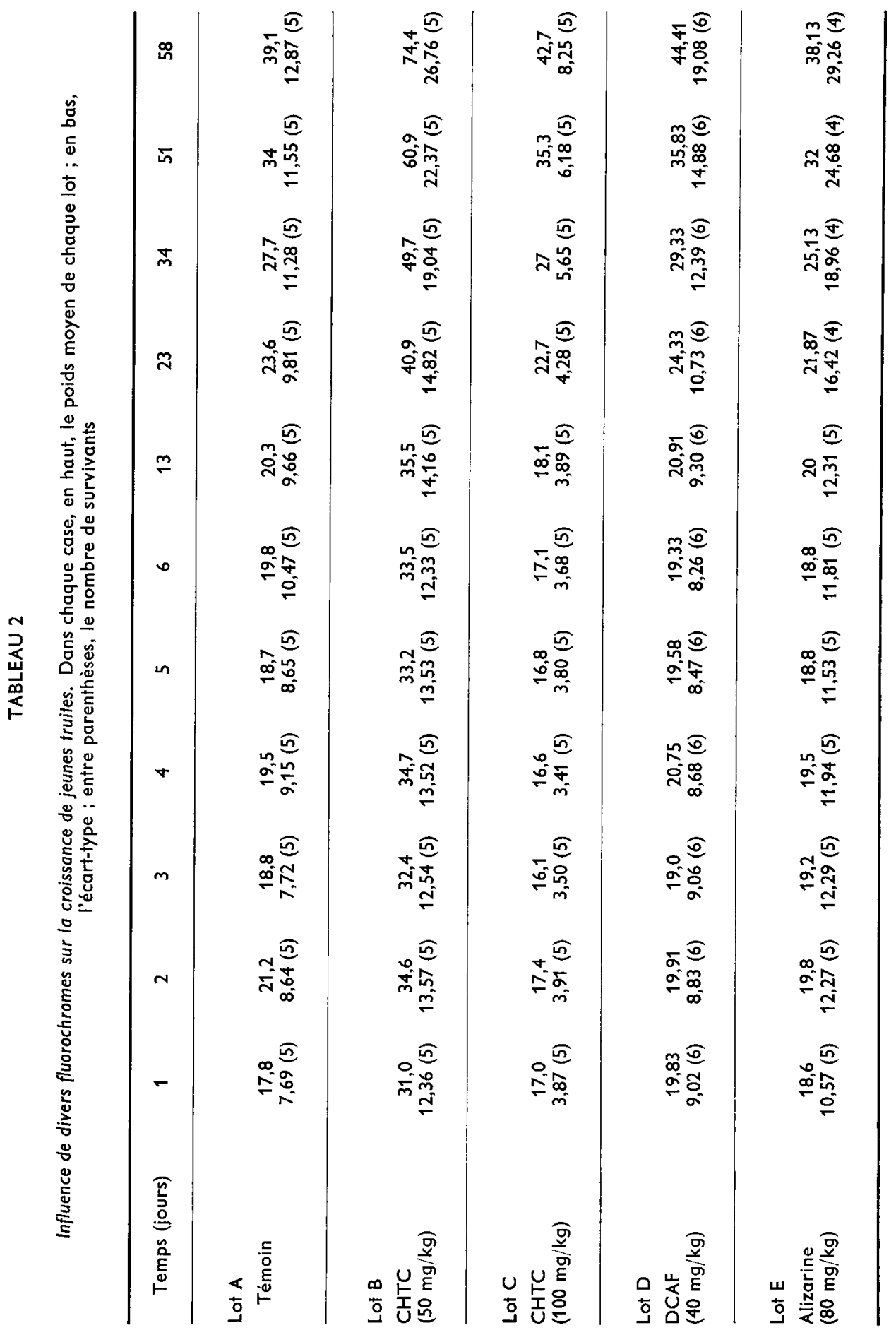


Des calculs statistiques ont éłé effectués sur les différents lots. Nous avons comparé les moyennes des poids des différents lots expérimentaux à celles des lots-témoins ef recherché leur intervalle de confiance pour de petits échantillons avec le test $t$ de Student. Nous avons adopté un coefficient de sécurité de 95 p. 100 . Toufefois dans les 4 lots affectés par la forte mortalité le petit nombre d'individus rescapés entraîne une augmentation de la variabilité et du degré de liberté, ce qui explique que certaines valeurs ne sont pas significatives malgré un fort éloignement des points sur les courbes des figures $3 a$ et $4 a$.

\section{Résultats.}

\section{1. - Action de la tétracycline.}

Nous n'avons pas observé de mortalité tant chez les truites (tabl. 2) que chez les carpes (tabl. 4) avec de faibles doses de CHTC $(50 \mathrm{mg} / \mathrm{kg}$ et $100 \mathrm{mg} / \mathrm{kg})$. Il n'existe pas de différences significatives entre l'évolution de la croissance pondérale de ces animaux et celle des témoins. Toutefois, chez les carpes injectées avec 50 ou $100 \mathrm{mg} / \mathrm{kg}$, la croissance pondérale est légèrement plus faible que celle des témoins; chez les truites, les croissances pondérales sont équivalentes (fig. 1 et 2 ). De plus, pendant les trois ou quatre premiers jours, on observe une stagnation ou une légère perte de poids des animaux injectés; mais rapidement la croissance pondérale reprend normalement

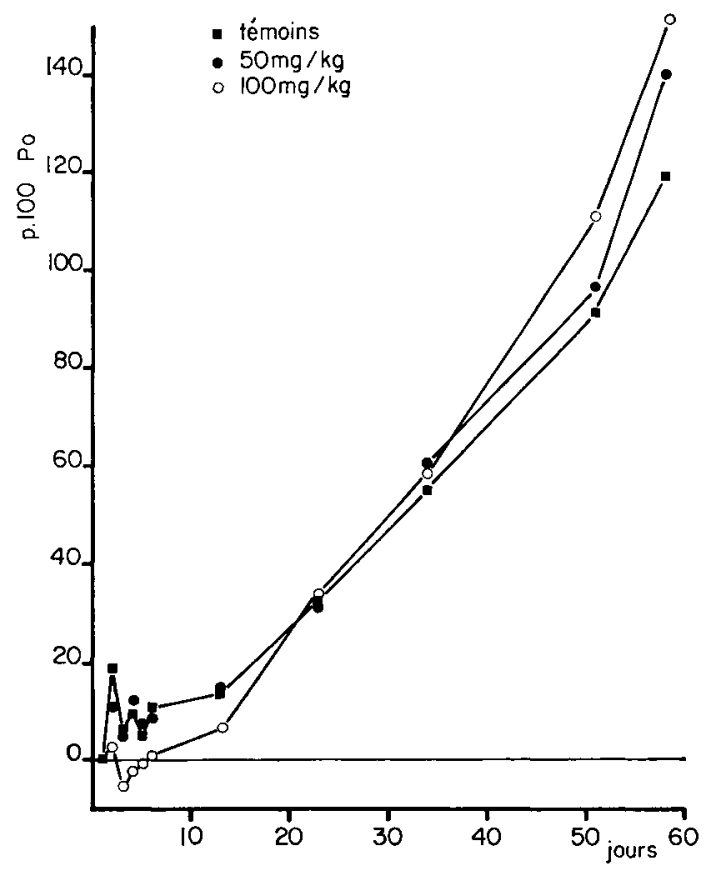

FIG. 1. - Action de faibles doses de CHTC sur la croissance pondérale (en p. 100 du poids initial moyen Po) de jeunes truites, en fonction du temps (en jours). 


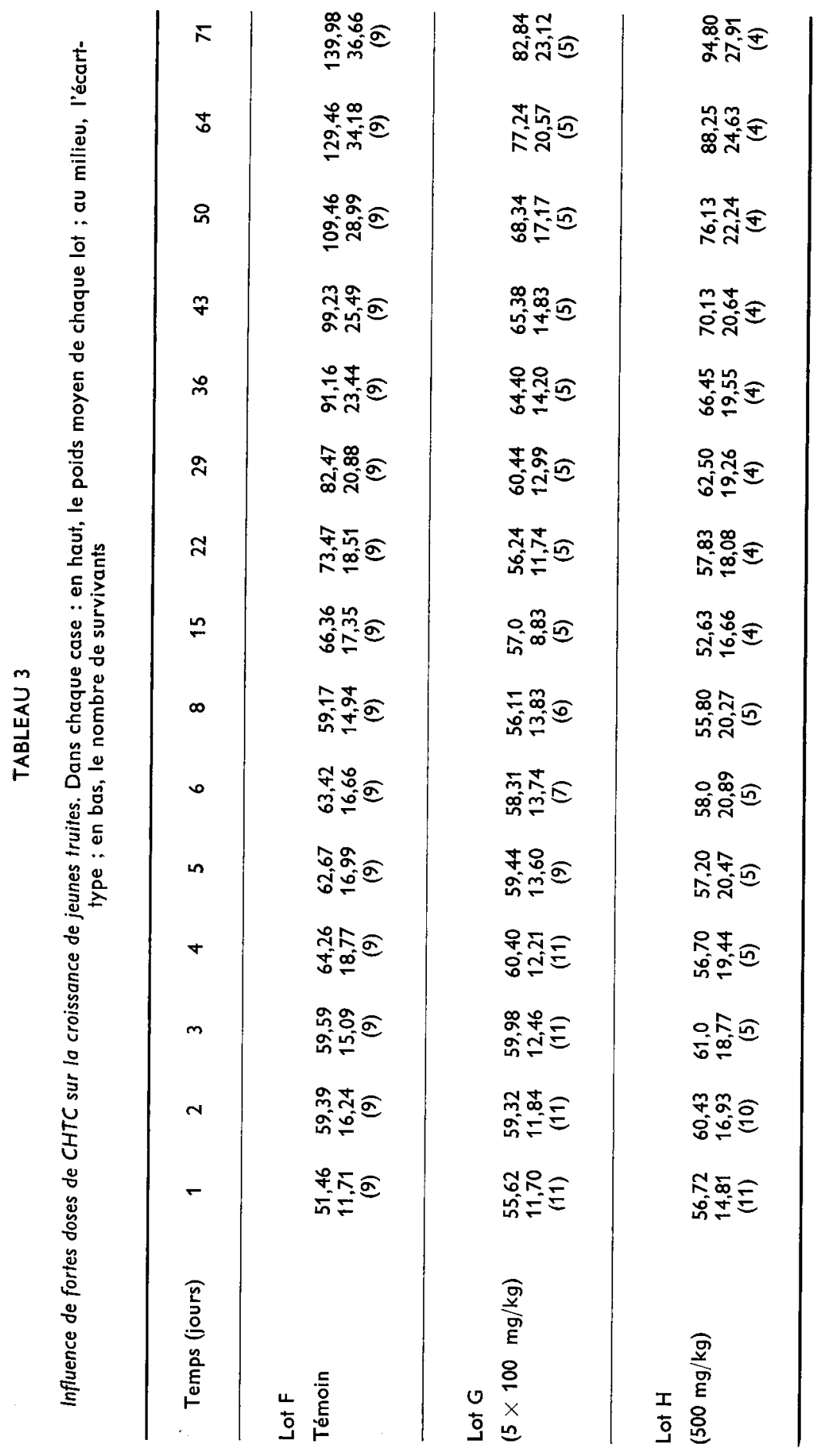


(fig. 1 et 2). Par contre les truites injectées avec la solution de $\mathrm{NaCl}$ ne présentent pas ce phénomène.

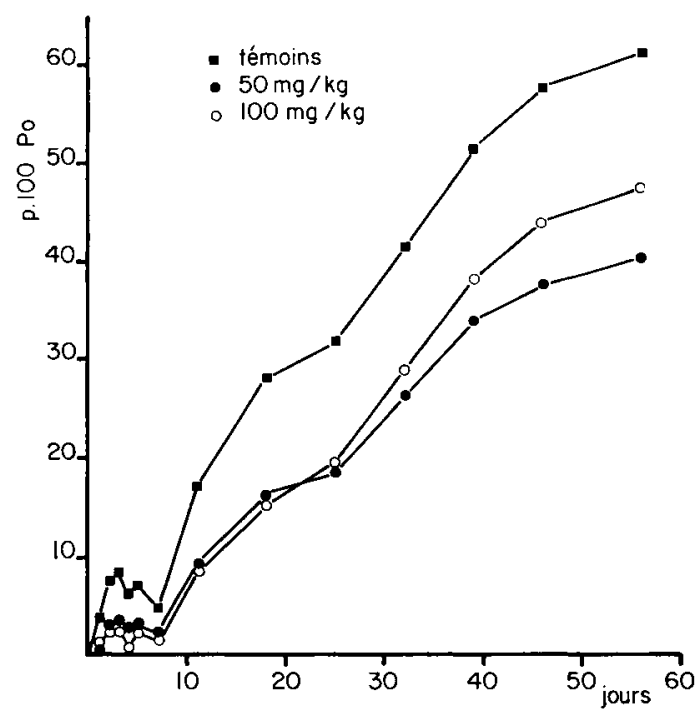

FIG. 2. - Action de faibles doses de CHTC sur la croissance pondérale (en p. 100 du poids initial moyen Po de jeunes carpes, en fonction du temps (en jours).

En revanche les fortes doses de CHTC $(5 \times 100 \mathrm{mg} / \mathrm{kg}$ et $500 \mathrm{mg} / \mathrm{kg})$ entraînent chez les carpes une mortalité importante au bout de la première semaine (tabl. 4) ; cette mortalité s'étale jusqu'à la fin du premier mois. Chez les truites, la mortalité esł encore plus précoce et se répartit entre les deuxième et quinzième jours qui suivent la première injection ( tabl. 3). Chez les truites et les carpes, on constate que la mortalité est plus importante pour une injection unique de $500 \mathrm{mg}$. Les animaux survivants reprennent leur croissance mais avec une vitesse moindre que chez les témoins ; les différences sont d'ailleurs significatives (fig. $3 a$ et $4 a$ ).

Des symptômes, différents chez les deux espèces, précèdent la mort des animaux : - chez la truite, présence d'une forte pigmentation sombre ; à l'autopsie, les viscères montrent un aspect normal ;

- chez la carpe, présence d'une hypervascularisation de la peau avec un ou plusieurs hématomes sous-cutanés plus ou moins étendus ; gonflement de la cavité abdominale et soulèvement des écailles. Les animaux présentent en outre une forte exophtalmie ef leurs parois musculo-cutanées sont chargées d'eau. L'examen des viscères montre un foie de couleur verte alors que les autres organes semblent intacts, au moins à l'œil nu. Dans les jours ou les heures qui précèdent la mort, des carpes présentent de nombreuses ulcérations allant jusqu'à la perforation des parois abdominales.

Chez les carpes rescapées, ces divers symptômes, tout en étant présents, s'avèrent moins graves et les ulcérations se cicatrisent. Mais le foie est encore vert au moment du sacrifice, en fin d'expérimentation (soit 56 jours après la première injection). II est à 


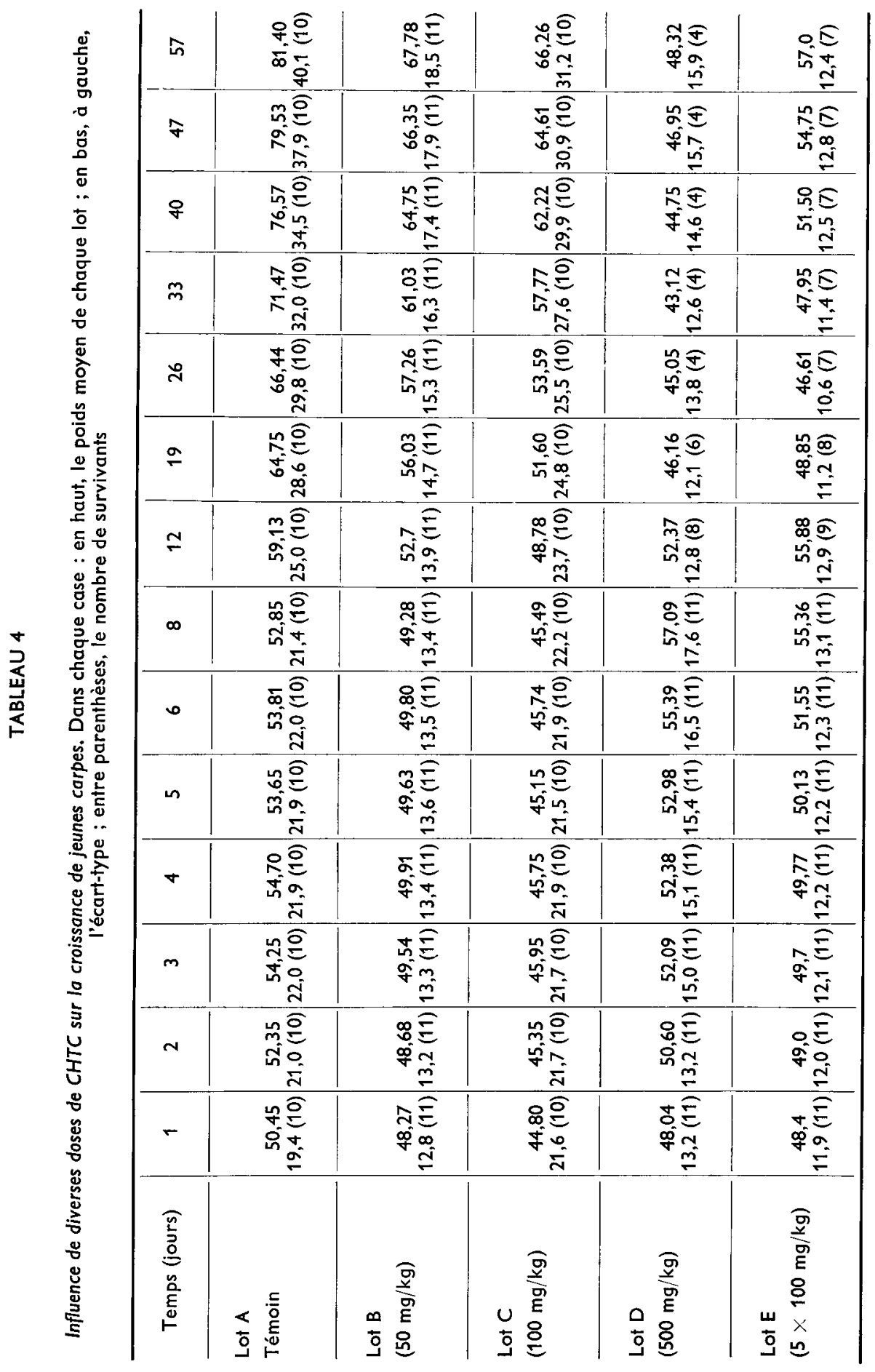


remarquer que les carpes qui ont reçu 50 ou $100 \mathrm{mg} / \mathrm{kg}$ de CHTC ont un foie de couleur brune normale.

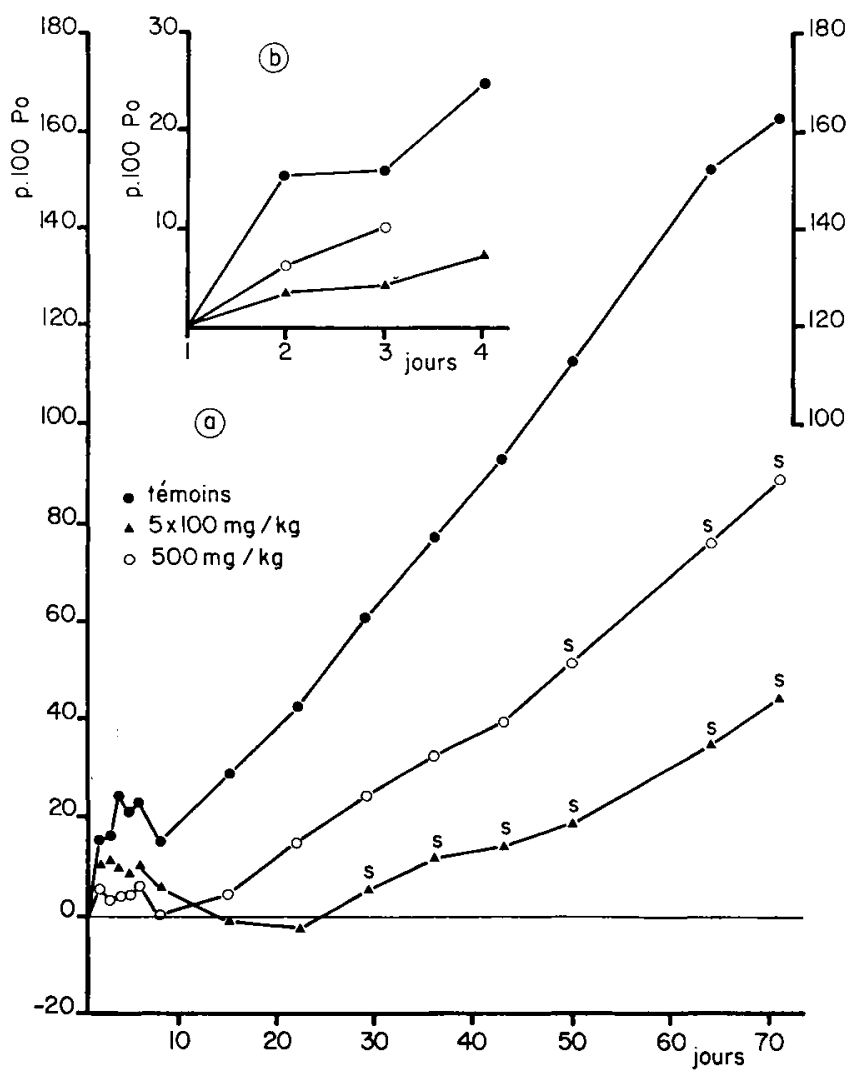

FIG. 3. - Action de fortes doses de CHTC sur la croissance pondérale (en p. 100 du poids initial moyen Po) de jeunes truites, en fonction du temps (en jours).

- Courbe $a$ : considère les animaux survivants (les points marqués d'un $\mathrm{S}$ sont significativement différents des témoins) ;

- Courbe $b$ : tient compte de tous les animaux, avant les premières mortalités.

Les deux courbes (fig. $3 b$ ef $4 b$ ) représentant la moyenne des croissances pondérales, respectivement chez la truite ef la carpe, pendant les premiers jours de l'expérimentation, montrent deux phénomènes différents. Chez les truites qui ont reçu les fortes doses de CHTC, la prise de poids est inférieure à celle des témoins alors que, au contraire, elle est beaucoup plus forte chez les carpes. Chez ces dernières, l'injection de $500 \mathrm{mg} / \mathrm{kg}$ de CHTC provoque une augmentation rapide du poids supérieure à celle des témoins. Chez celles qui ont reçu la même quantité de fluorochrome en 5 injections, cette augmentation, d'abord faible, s'accroît intensément dès le $4^{\mathrm{e}}$ jour ; un effet cumulatif se fait alors sentir. Compte tenu des symptômes observés et de l'action des faibles doses de CHTC chez la carpe, nous pensons qu'il doit se produire une absorption ou/et une rétention d'eau, ce que confirme les deux expériences complémentaires. 


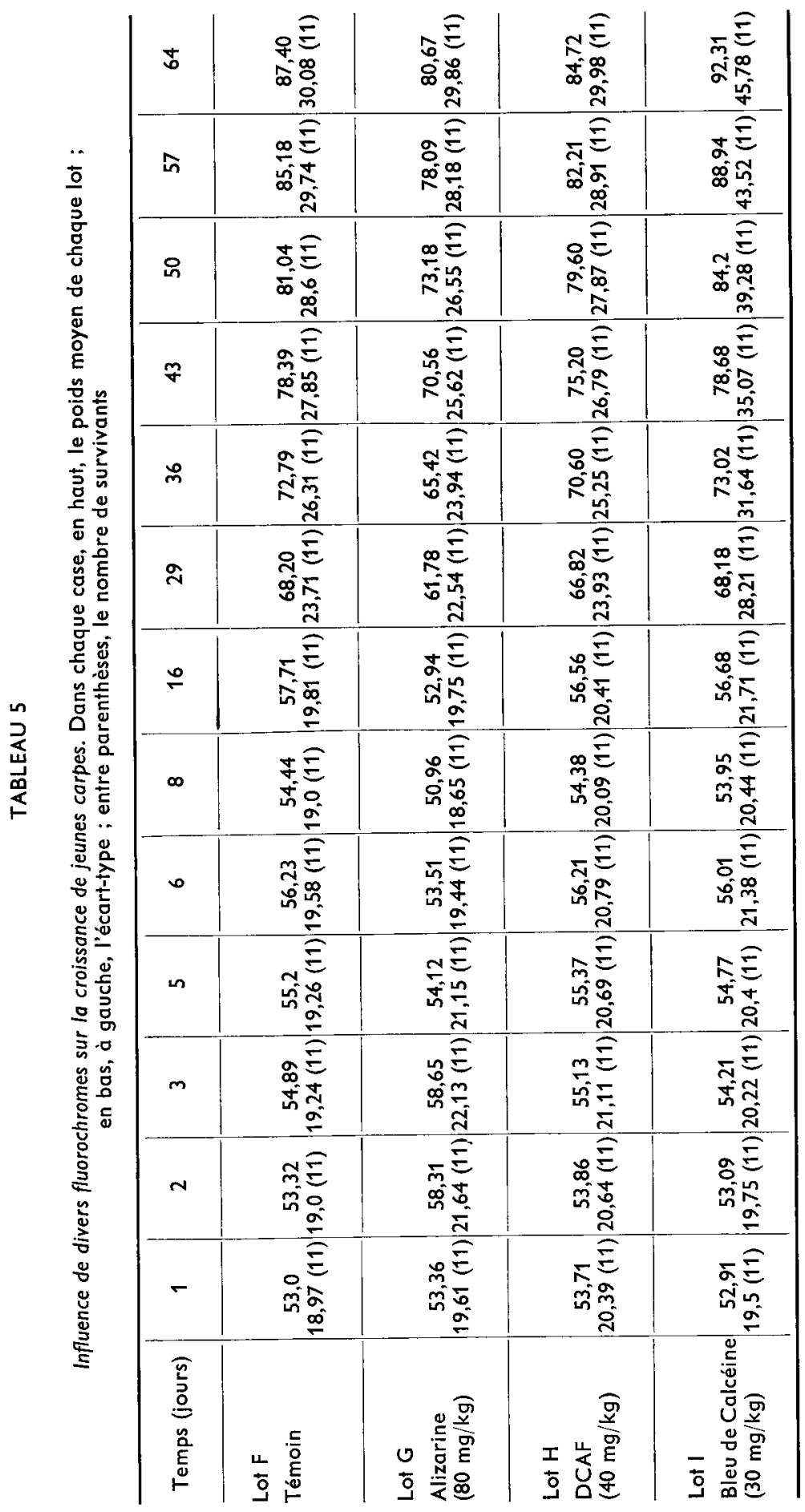


En effet les deux carpes qui ont reçu $500 \mathrm{mg} / \mathrm{kg}$ et qui sont maintenues à jeun présentent une assez forte augmentation de leur poids, non due à l'alimentation : 27 p. 100 pour la première en $48 \mathrm{~h}$ et $25 \mathrm{p} .100$ pour la deuxième en $72 \mathrm{~h}$. La mort de ces deux animaux a été trop rapide pour voir apparaître les symptômes décrits plus haut. D'autre part, les deux carpes injectées avec du $\mathrm{NaCl}$ onf été sacrifiées au bout de trois semaines dans un excellent état morphologique alors que les deux ayant reçu les $500 \mathrm{mg} / \mathrm{kg}$ sont mortes quelques jours seulement après l'injection, après avoir manifesté une forte exophtalmie et un soulèvement des écailles ; la cavité abdominale était gonflée, les muscles chargés d'eau et le foie avait la couleur verte signalée précédemment. Elles présentaient de plus une vivacité moindre avec une prise de poids respectivement de 25 p. 100 et 22 p. 100 alors que les deux carpes qui ont été traitées avec le $\mathrm{NaCl}$ ont pris, dans le même temps, 2,4 p. 100 et 5,8 p. 100. Ces deux dernières n'ont pas présenté de baisse du poids corporel pendant les premiers jours de l'expérimentation.

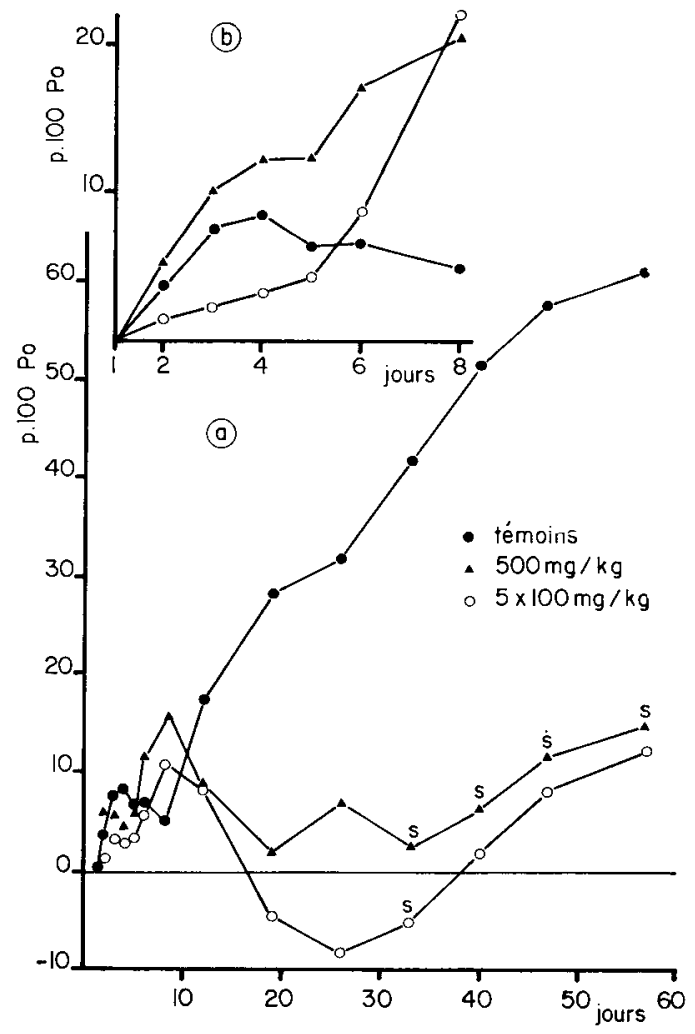

FIG. 4. - Action de fortes doses de CHTC sur la croissance pondérale (en p. 100 du poids initial moyen Po) de jeunes carpes, en fonction du temps (en jours).

- Courbe a : considère les animaux survivants (les points marqués d'un $S$ sont significativement différents des témoins).

- Courbe $b$ : tient compte de tous les animaux, avant les premières mortalités. 
2. - Action des autres fluorochromes.

La fluorescéine et le bleu de calcéine n'ont pas d'effet sur la vie des animaux et n'ont d'action significative ni sur la croissance pondérale ni sur la taille des truites ou des carpes (fig. 5). Si l'alizarine se montre sans action chez les carpes (tabl. 5), elle a peut-être provoqué la morf d'une truite (tabl. 2) ; toutefois la prise de poids des survivants n'esł pas significativement différente de celle des témoins. Comme pour les faibles doses de fétracycline, il y a, pendant les premiers jours, une stagnation de la croissance pondérale chez les animaux injectés (fig. 5).

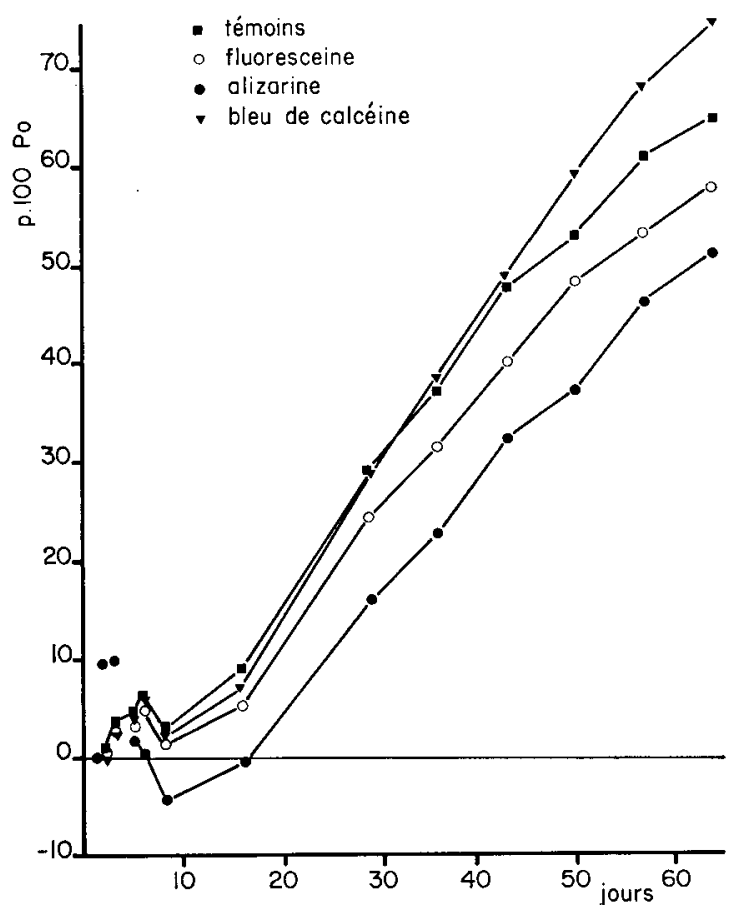

FIG. 5. - Action de divers fluorochromes (autres que le CHTC) sur la croissance pondérale (en p. 100 du poids initial moyen Po) de jeunes carpes, en fonction du femps (en jours).

Le bleu de calcéine présente un intérêt limité dans la mesure où la fluorescence des tissus squelettiques marqués disparaît très vite pendant l'observation, probablement par action des rayons UV. Rahn et Perren (1970) avaient déjà remarqué ce phénomène.

\section{Discussion.}

Nous avons montré que l'administration de faibles doses de fluorescéine, d'alizarine ou de bleu de calcéine, n'était pas toxique pour les poissons ef qu'elle n'influençait pas significativement leur croissance pondérale. Ces résultats confirment ceux obtenus chez les Mammifères pour des doses équivalentes de fluorochromes (Solheim, 1974 ; 
Vilmann, 1974 ; Rasmussen, 1975). Malgré tout Solheim (1974) et Vilmann (1974) signalent que des doses égales ou supérieures à $100 \mathrm{mg} / \mathrm{kg}$ d'alizarine entraînent chez les rats une diminution passagère ou prolongée du gain de poids. Bien que la dose d'alizarine que nous employons soit proche de celle définie par ces auteurs, nous n'avons pas observé une telle diminution significative du gain de poids.

De même, à doses faibles (50 ou $100 \mathrm{mg} / \mathrm{kg}$ ) le CHTC n'est pas toxique et il ne modifie pas la croissance pondérale de nos poissons. Compte tenu de quelques travaux antérieurs (Weber et Ridgway, 1962 ; Meunier et Boivin, 1974), nous confirmons donc que les doses de $50 \mathrm{mg} / \mathrm{kg}$ de CHTC sont suffisantes pour obtenir un bon marquage du tissu osseux des poissons téléostéens. Pendant les 3 ou 4 jours qui suivent l'injection, on note une légère chute de poids, non significative, qui est probablement due à la baisse de la prise de nourriture et non pas au mode opératoire. Cette perte de poids disparaît très vite et, en fin d'expérience, au bout de deux mois en moyenne, les différences en plus ou en moins, par rapport aux témoins, sont minimes et non significatives.

En ce qui concerne l'action des fortes doses de tétracycline, nos résultats sont intéressants quant aux effets provoqués chez les poissons. Chez la truite, tout d'abord, ces fortes doses de CHTC provoquent une mortalité importante et rapide, sans que l'on puisse toutefois observer d'évolution pathologique spectaculaire; cette espèce apparaît donc très sensible aux fortes doses de CHTC. Par contre, chez la carpe, des symptômes pathologiques variés sont observés et, si la mortalité est aussi importante que chez la truite, son évolution est plus étalée dans le temps. De plus, nous avons observé que les animaux survivants présentaient un retard dans leur croissance pondérale. Cette dernière observation est à rapprocher de celles faites, lors de l'administration de fortes doses de tétracycline, chez l'embryon de poulet (Bevelander et al., 1959) et chez les rats embryonnaires ou nouveaux-nés (Bevelander, 1964 ; Marois et Marois, 1971).

Chez la carpe, la physiologie du foie paraît fortement perturbée par des doses importantes de tétracycline. Or, chez la souris, Böttiger (1955) a constaté que le foie était l'organe présentant la plus forte concentration et la plus longue rétention de tétracycline et que ceci pouvait entraîner des complications hépatiques chez ces animaux. Les observations de cet auteur sont donc à rapprocher des nôtres. Par ailleurs, les fortes doses de tétracycline provoquent une absorption et/ou une rétention d'eau, comme le montrent nos deux expériences complémentaires (animaux à jeun et injection d'un volume équivalent de $\mathrm{NaCl}$ à 0,6 p. 100). II serait nécessaire d'effectuer une étude de l'excrétion urinaire pour pouvoir vérifier cette hypothèse. En tout état de cause les grandes quantités de CHTC exercent une influence néfaste sur la balance hydrique de ces animaux. La présence des ulcérations sur les flancs des carpes pourrait s'interpréter comme l'apparition de souches de parasites pathogènes rendus résistants à l'antibiotique (Christensen, 1966). Quant à l'exophtalmie elle semble refléter à la fois le mauvais état général des animaux et l'hydratation des tissus.

Chez la truite, par contre, il est beaucoup plus difficile d'émettre une hypothèse quant à l'action de la tétracycline, en l'absence de véritables symptômes avant la mort des animaux.

Il reste à envisager maintenant le problème de l'action locale des fluorochromes se fixant dans les tissus squelettiques. En effet, nous ne pouvons pas éliminer, dans l'état actuel de nos recherches, l'existence d'une action locale de ces marqueurs, à l'emplacement de leur dépôt, sur l'ostéogenèse, sur les processus de minéralisation, sur la 
phase minérale du dépôt osseux et/ou sur la trame organique de l'os, ainsi que sur les cellules qui la sécrètent. C'est ainsi que Bevelander et Goss (1962) puis Bevelander (1964) mentionnent une inhibition de la calcification et de l'ossification sous l'influence des tétracyclines. Des travaux récents, réalisés chez quelques Mammifères, ont montré que des doses relativement faibles de diverses sortes de tétracyclines, injectées en une seule fois ou de façon répétitive, peuvent entraîner un certain nombre de malformations des tissus squelettiques.

L'émail paraît particulièrement sensible et des défauts dans son développement sont mentionnés par différents auteurs (Löfgren et al., 1968 ; Nylen et al., 1972 ; Omnell et al., 1970 ; Westergaard et Nylen, 1975) ; ces défauts ont une ampleur proportionnelle à la dose injectée. Ceci est confirmé par Kruger (1975) qui décrit, en fonction des doses injectées, l'évolution des changements dans la minéralisation de la dentine et des altérations ultrastructurales des odontoblastes et des adamantoblastes. Notons que l'emploi expérimental des tétracyclines à doses pharmacologiques chez les Téléostéens pourrait peut-être permettre de résoudre un certain nombre de questions liées au problème de l'opposition entre «émail vrai » et « tissu adamantoïde » (Bergot, 1975). En effet, l'« émail vrai » (d'origine ectodermique) et les adamantoblastes paraissent beaucoup plus sensibles que les odontoblastes à l'action locale des tétracyclines.

En ce qui concerne l'os, Simmons et al. (1977) ont montré que la tétracycline pouvait causer des changements dans l'activité d'une population ostéoblastique. Par ailleurs, la croissance du tissu osseux du singe Rhesus peut, dans certains cas, être ralentie (Yen et Shaw, 1972, 1975) alors que la dentinogenèse ne semble pas affectée (Yen et Shaw, 1974). D'autres études au contraire ne montrent pas d'influence de doses assez faibles de tétracycline ef de fluorescéine sur la formation et la minéralisation de la dentine chez le rat (Solheim, 1974 ; Rasmussen, 1975).

En fait, devant la diversité de ces observations, nous rejoignons les conclusions exprimées dans la revue de Skinner et Nalbandian (1975) en disant que les fluorochromes et surtout les tétracyclines ont une action sur les tissus squelettiques, qui dépend des espèces, de l'âge des animaux (Vilmann, 1974 ; Westergaard et Nylen, 1975), de la nature des tissus récepteurs, des quantités injectées (Kruger, 1975 ; Westergaard et Nylen, 1975) et des modes d'administration. II faut remarquer, en outre, qu'à l'intérieur des tétracyclines, les effets néfastes varient avec les différentes substances (Yen et Shaw, 1974). Il est également important de rappeler que les tétracyclines affectent les phénomènes de minéralisation in vitro, comme Kaitila (1971) l'a démontré, el cet auteur envisage des mécanismes identiques lors de l'ostéogenèse ef de la formation des dents in vivo. Par ailleurs, Halme et al. (1969) ont montré un effet inhibiteur de la tétracycline sur la biosynthèse du collagène et sur la minéralisation, dans des cultures d'os d'embryons de souris.

Cet ensemble de résultats, obtenus essentiellement chez des Mammifères, nous incite à penser que ce travail sur la toxicité des fluorochromes chez les poissons doit être prolongé et approfondi par : 1) l'étude ultrastructurale et biophysique des tissus squelettiques en cours de minéralisation pendant l'administration des tétracyclines; 2) l'éfude quantitative de cette minéralisation. Ces réserves étant faites, le présent travail démontre qu'en pratique les fluorochromes, administrés à doses convenables chez les Poissons, marquent leurs tissus squelettiques sans entraîner de séquelles phy- 
siologiques indésirables. Comme l'intérêt de la technique du marquage vital dans diverses études biologiques chez les Poissons (écologie, démographie...) n'est plus à démontrer, nous possédons bien là une technique très avantageuse, à condition toutefois de respecter les doses optimales pour avoir un bon marquage compatible avec la croissance et la vie normale des animaux.

Reçu en février 1978.

Accepté en juin 1978.

Remerciements. - Les auteurs expriment leur gratitude aux Professeurs C. A. Baud et $Y$. François et à MM. J. Castanet et $A$. de Ricqles pour leurs suggestions ef leurs critiques au cours de la préparation de ce manuscrit. Ils remercient vivement les Etablissements Spécia qui leur ont fourni gracieusement le chlorhydrate de tétracycline utilisé dans ce travail.

\section{Références}

BERGOT C., 1975. Morphogenèse et structure des dents d'un téléostéen (Salmo fario L.). J. Biol. Buc., 3, 301-324.

BEVELANDER G., 1964. The effect of tetracycline on mineralization and growth. Adv. oral. Biol., 1, 205-223.

BEVELANDER G., GOSS R. J., 1962. Influence of tetracycline on calcification in normal and regenerating teleost scales. Nature, 193, 1098-1099.

BEVELANDER G., NAKAHARA H., ROLLE G. K., 1959. Inhibition of skeletal formation in the chick embryo following administration of tetracycline. Nature, 184, 728-729.

BONT A. F. de, VAN COILLIE R., 1966. Scalimétrie à l'aide de tétracyclines. Verh. int. Ver. Limnol., 16, 1130-1134.

BÖTTIGER L. E., 1955. On the distribution of chlortetracycline in the body. Acta med. scand., 151, 343-348.

CHRISTENSEN N. O., 1966. Maladies des Poissons. Ed. franç., Synd. Pisc. Salm. Fr., Paris, Pp. 97.

DHEM A., 1967. Le remaniement de l'os adulfe. Arscia, Bruxelles, pp. 118.

DUPONT H., 1964. Confribution à l'étude de la croissance et du remaniement osseux chez la souris. Rev. suisse Zool., 71, 709-775.

HALME J., KIVIRIKKO K. I., KAITILA I., SAXEN L., 1969. Effect of tetracycline on collagen biosynthesis in cultured embryonic bones. Biochem. Pharmacol., 18, 827-836.

HUET M., 1962. Qualités des eaux à exiger pour le poisson. Bull. Inform. F. E. P. E., 6, 3-11.

JONES I. W., 1969. Notes on marking salmon fingerlings with tetracyclines. Ann. Progr. Rept. Oregon, Fish. Com., 6 pp.

KAITILA I., 1971. The mechanism by wich tetracycline hydrochloride inhibits mineralization in vitro. Biochem. biophys. Acta, 244, 584-594.

KOBAYASHI S., YUKI R., FURUI T., KOSUGIYAMA T., 1964. Calcification in fish and shellfish. I. Tetracycline labeling patterns of scale, centrum and otolith in young glodfish. Bull. jap. Soc. sci. Fish., 30, 6-13.

KRUGER B. J., 1975. Dose dependent ultrastructural changes induced by tetracycline in developing dental tissues of the rat. J. dent. Res., 54, 822-828.

LÖFGREN C. G., OMNELL K. A.., NYLEN M. U., 1968. Effect of intraperitoneal injections of tetracycline hydrochloride and oxytetracycline on forming enamel of rat incisors. Calc. Tiss. Res., 2, 145-156.

LOPEZ E., 1970. L'os cellulaire d'un poisson téléostéen Anguilla anguilla L. I. - Etude histocytologique et histophysique. Z. Zellforsch., 109, 552-565.

MAROIS P., MAROIS M., 1971. Action des tétracyclines sur la dent et sur l'os. Biol. Méd., 60, $293-362$.

MEUNIER F., 1972. Marquages simples ef multiples du tissu osseux de quelques Téléostéens par des substances fluorescentes. C. R. Acad. Sci. Paris, sér. D, 275, 1685-1688. 
MEUNIER F., 1974. La technique de marquage vital des tissus squelettiques des poissons. Bull. Fr. Pisc., 255, 51-57.

MEUNIER F., BOIVIN G., 1972. Marquages multiples du tissu osseux de quelques Téléostéens à l'aide de plusieurs fluorochromes. Bull. Soc. Zool. Fr., 97, 539-540.

MEUNIER F., BOIVIN G., 1974. Divers aspects de la fixation du chlorhydrate de tétracycline sur les tissus squelettiques de quelques Téléostéens. Bull. Soc. Zool. Fr., 99, 495-504.

MILCH R. A., RALL D. P., TOBIE J. E., 1957. Bone localization of the tetracyclines. J. nat. Cancer Inst., 19, 87-91.

NYLEN M. U., OMNELL K. Å., LÖFGREN C. G., 1972. An electron microscopic study of tetracyclineinduced enamel defects in rat incisor enamel. Scand. J. dent. Res., 80, 384-409.

OLERUD S., LÖRENZI G. L., 1970. Triple fluorochrome labeling in bone formation and bone resorption. J. Bone Joint Surg., 52-A, 274-278.

OMNELL K. A., LÖFGREN C. G., NYLEN M. U., 1970. Tetracycline-induced enamel defects in the rat incisor. Arch. oral Biol., 15, 645-661.

RAHN B. A., PERREN S. M., 1970. Calcein blue as a fluorescent label in bone. Experientia, 26, 519.

RASMUSSEN P., 1975. Effect of oxytetracycline and purified calcein (DCAF) on the apposition and mineralization of rat incisor dentin. Scand. J. dent. Res., 83, 233-237.

SIMMONS D. J., SIMMONS N. B., MARSHALL J. H., 1970. The uptake of calcium-45 in the acellularboned toadfish. Calc. Tiss. Res., 5, 206-221.

SIMMONS D. J., BATES M. L., TEITELBAUM S. L., 1977. Tetracycline-induced phase shift in osteogenic cell populations. Anat. Rec., 187, 713-714.

SKINNER H. C. W., NALBANDIAN J., 1975. Tetracyclines and mineralized tissues : review and perspectives. Yale J. Biol. Med., 48, 377-397.

SOLHEIM T., 1974. Pluricolor fluorescent labeling of mineralizing tissue. Scand. J. dent. Res., 82, 19-27.

SUZUKI H. K., MATHEWS A., 1966. Two color fluorescent labeling of mineralizing tissues with tetracycline and 2-4-bis (N-N' Dicarbomethylaminomethyl) fluorescein. Stain Techn., 41, 57-60.

VAN COILLIE R., 1967. Etude à l'aide de tétracyclines de la croissance périodique des écailles de Téléostéens. Nat. Can., 94, 29-58.

VILMANN H., 1974. The effects of alizarin red $S$ on weight gain and skull growth in the rat. Arch. oral Biol., 19, 1007-1010.

WEBER D. D., RIDGWAY G. J., 1962. The deposition of tetracycline drugs in bone and scales of fish and its possible use for marking. Progr. Fish. Cult., 24, 150-155.

WEBER D. D., RIDGWAY G. J., 1967. Marking pacific salmon with tetracycline antibiofics. J. Fish. Res. Con., 24, 849-865.

WESTERGAARD J., NYLEN M. U., 1975. Dose and age dependent variations in effect of fetracycline on enamel formation in rat. Scand J. dent. Res., 83, 209-232.

YEN P. K. J., SHAW J. H., 1972. Preliminary study o-inhibitory effects of tetracyclines on membranous bone growth in Rhesus monkeys. J. dent. Res., 51, 1651-1657.

YEN P. K. J., SHAW J. S., 1974. Effects of tetracyclines on membranous bone growth and dentin apposition in young Rhesus monkeys. J. dent. Res., 53, 897-906.

YEN P. K. J., SHAW J. H., 1975. Effects of repeated oral doses of Demethylchlortetracycline on bones and dentin of young Rhesus monkey. J. dent. Res., 54, 358-364. 